\title{
Mortality associated with tuberculosis/HIV co-infection among patients on TB treat- ment in the Limpopo province, South Africa.
}

\author{
Tiyani. E Mabunda ${ }^{1}$, Nalezani J Ramalivhana ${ }^{2}$,Yoswa M Dambisya ${ }^{3}$
}

1. Limpopo Provincial Department of Health, Polokwane 0700; South Africa

2. Department of Public Health, University of Limpopo, Sovenga 0727, South Africa.

3. Department of Pharmacy, University of Limpopo, Sovenga 0727, South Africa.

\begin{abstract}
Background: South Africa has a high tuberculosis burden, and Limpopo Province experienced higher than national average TB mortality rates between 1997 and 2008.

Objective: To establish factors associated with TB mortality in Limpopo Province in 2008.

Design: Retrospective study using provincial data for patients who died after commencing TB treatment between 01 January 2008 and 31 December 2008.

Results: In 2008, some 18074 patients started treatment: 15995 (88.5\%) had pulmonsry TB (PTB), while 2079 (11.5\%) had Extra pulmonary TB (EPTB). Overall, 2242 (12.4\%) patients died, mainly PTB patients ( $\mathrm{n}=1906$;

$85 \%$ ), more males $(\mathrm{n}=1159,51.7 \%)$, mainly those aged 25 to 54 years $(\mathrm{n}=1749,78.0 \%)$, and new cases $(1914 ; 85.4 \%)$. TB mortality was significantly higher among smear negative than smear positive patients $(17 \%$ vs $13.8 \%$; $<<0.001)$, among those with EPTB compared to PTB patients $(\mathrm{P}<0.001)$, and among re-treatment cases $(\mathrm{P}<0.001)$. Only 4237 $(23.4 \%)$ patients had HIV status known, with higher mortality found among HIV positive than the HIV negative patients $(\mathrm{P}<0.0001)$; but HIV status was not known for the majority who died $(\mathrm{n}=1685,75.2 \%)$.

Conclusion: Higher mortality was associated with age 22-55 years; smear negativity, EPTB, HIV infection, and re-treatment. The findings call for greater integration of TB control efforts and HIV services, especially among the 22-55 year age group.
\end{abstract}

Keywords: Tuberculosis/HIV, Limpopo, South Afric

DOI: http://dx.doi.org/10.4314/ahs.v14i4.12

\section{Introduction}

South Africa was ranked fifth among the 22 tuberculosis (TB) high burden countries in 2007 and third after India and China among the 22 countries carrying $82 \%$ of global TB burden in $2010^{1}$. According to the World Health Organization (WHO) Global TB Report 2009, South Africa had nearly 460000 new TB cases in 2007 with an incidence rate of 948 cases per 100000 population, and a TB prevalence rate of 692 per 100 000 population per year ${ }^{2}$. South Africa has the largest number of people living with human immunodeficiency virus (HIV) and acquired immune deficiency syndrome (AIDS), as a result HIV associated TB has become a major clinical and public health problem ${ }^{3}$.
Corresponding author:
Yoswa Dambisya
Department of Pharmacy
School of Health Sciences University of
Limpopo Private Bag X1106
Sovenga 0727, South Africa
E-mail: yoswad@gmail.com

The (HIV) epidemic that has progressed in tandem with TB infection poses a big threat to TB Control effort $^{4,5}$.

Limpopo is one of the poorest provinces of South Africa, with relatively poor infrastructure and public services, including health. The province has a vibrant TB Control Programme that works according to the national guidelines, including the capture of TB statistics on the electronic database, ETR.Net. Programmatic data shows that the recorded number of TB cases in the Limpopo Province increased more than threefold from 6286 in 2000 to 22836 in 2009 . However, the bacteriological coverage decreased from $90.7 \%$ to $79.9 \%$ over the same period. At the same time, the death rate in new smear positive patients in the province was consistently higher than the national average between 1997 and 2008.

The Desmond Tutu TB Centre, University of Stellenbosch, in collaboration with the National Department of Health, is coordinating efforts to strengthen the capacity of the provinces to conduct operations re- 
search on TB. In this context, the Limpopo Province Statistical analysis

team undertook a study on the high death rates among Summary statistics were generated using Microsoft ExTB patients on treatment, with a view to contribute to cel, while SPSS version 20 was used for further analysis understanding the factors responsible for the high TB of the data for association between various variables mortality rate in the province. "Died" was taken to re- and TB mortality. The association between variables fer to a TB client who died from any cause during treat- and mortality was tested using the Chi-square test and ment, according to $\mathrm{WHO}$ guidelines. The aim of the post hoc Bonferroni's correction, with $\mathrm{P}<0.05$ as the study was to assess the factors associated with mortality limit of significance.

among TB patients who started treatment between 01 January and 31 December 2008.

\section{Study design}

Methodology

This was a quantitative, retrospective study using ETR Net provincial data for patients who initiated TB treatment from 1 January 2008 to 31 December 2008 The data were imported from ETR.net to Microsoft Excel, using a data capture template that was developed for that purpose. The data were then analysed to establish the TB mortality for the year, and the characteristics of patients who died.

\section{Study population}

All TB patients who died after starting treatment from

1 January until 31 December 2008 were included in the study.

\section{Data collection}

The age and sex of patients that died after commencement of treatment in 2008 were recorded. The association between smear positivity and outcome were analysed using a $2 \mathrm{X} 2$ table matching smear positivity and outcome. Where the diagnosis was confirmed by culture, that was recorded; and where sensitivity was tested, the results were captured as well, including mention of MDR/XDR TB where applicable. The HIV status was recorded as HIV positive, HIV negative or no HIV test done, and among those who were HIV positive, any additional information, such as CD4 cell count, and whether or not they were on HAART was also noted. The site of disease was recorded as pulmonary, extra-pulmonary, or both; while the diagnostic category was recorded as new patient or retreatment, and for retreatment this was noted as after default or relapse, or some other form of treatment failure.

\section{Ethical considerations}

The study protocol was approved by the University of Limpopo Polokwane-Mankweng Research Ethics Committee. In addition, permission was obtained from the Limpopo Province Department of Health and Social Development Research Committee, and the proposal was further reviewed and approved by the International Union against TB and Lung Diseases (IUATLD) prior to commencement of the study. This was a non-intrusive study that utilized retrospective data; . All data captured were without specific patient identifiers, to ensure the anonymity of the patients and all the information obtained was treated with utmost confidentiality.

\section{Results}

A total of 18074 patients $(52.3 \%$ male $)$ started treatment during the year 2008; . Of those 15995 (88.5\%) had pulmonary TB, while $2079(11.5 \%)$ had extra pulmonary TB; $16013(88.6 \%)$ were new cases, $664(5.3 \%)$ were relapses, $622(3.4 \%)$ had previously deand $187(1.0 \%)$ were "all other retreatment" cases. The number of deaths recorded for the period unde review was $2242(12.4 \%)$, being $1083(48.3 \%)$ female and $1159(51.7 \%)$ male, with no gender-related difference in TB mortality.

As shown in Figure 1, most of those who died $(78 \%)$ were aged 25 to 54 years, while Figure 2 shows that increasing age was associated with increased likelihood of death for TB patients on treatment $(\mathrm{X} 2=$ 180.1; $\mathrm{df}=5 ; \mathrm{P}<0.0001$ ).

Figure 1: The respective figures were $94(4.2 \%)$ patients were aged 4 years and below, $42(1.9 \%)$ were aged 5 to 14 years, $116(5.2 \%)$ were aged $15-24$ years, 1652 $(78.0 \%)$ were 25 to 54 years, $281(12.5 \%)$ were 55 to 74 years, and $57(2.5 \%)$ were older than 75 years. faulted, $288(1.6 \%)$ had a history of treatment failure,
Figure 1: Ago Range (Years) Distribution of Pationts who died in 2008

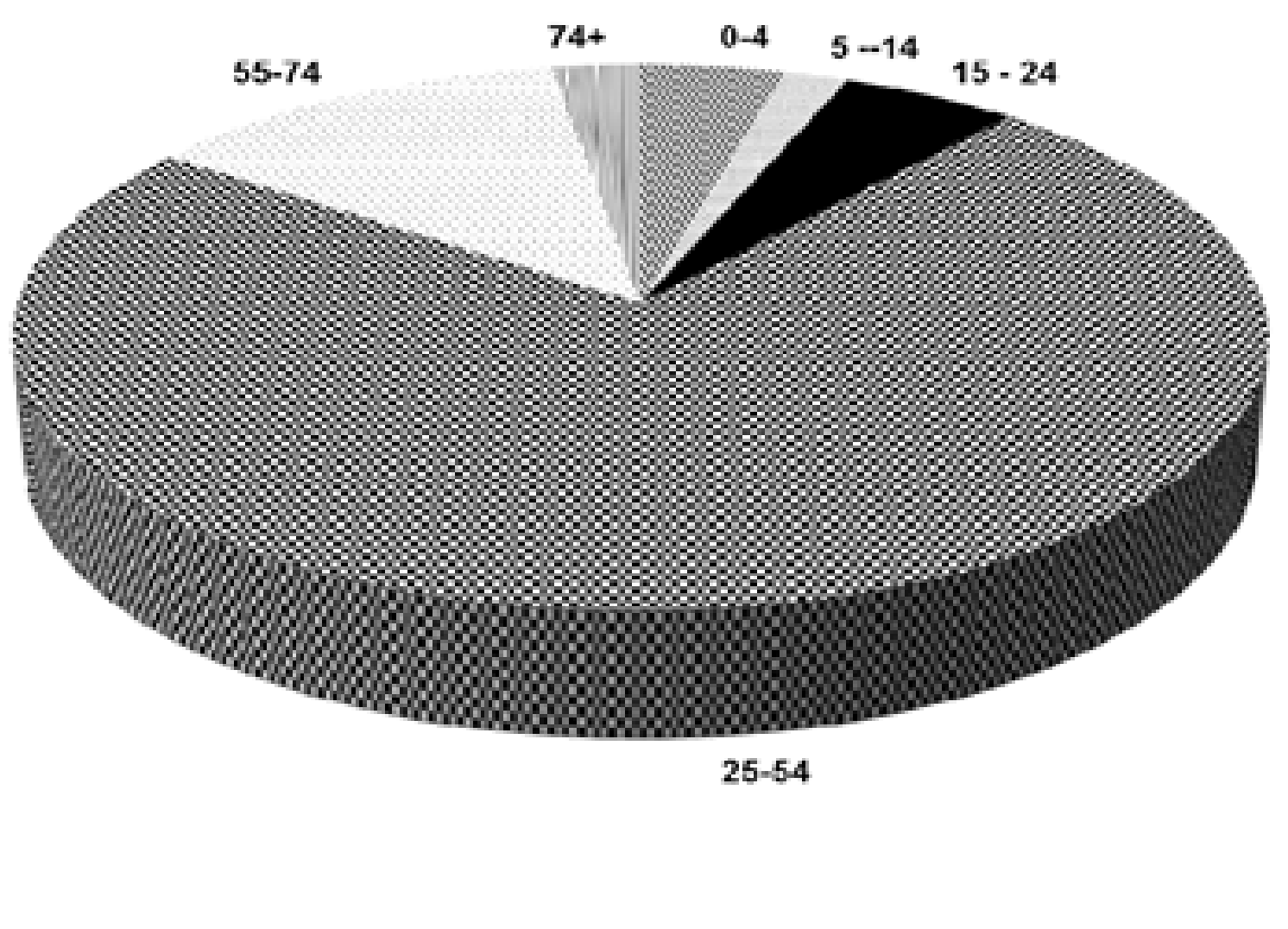

New cases accounted for $85.4 \%(\mathrm{n}=1914)$ of total mortality, while 146 (6.5\%) deaths were relapsed PTB cases, $104(4.6 \%)$ were those who had previously defaulted treatment; $45(2.0 \%)$ had history of treatment failure; and $31(1.4 \%)$ were other retreatment cases. Proportionately, $12 \%$ of the new cases died compared to $15.1 \%$ of the relapse cases, $16.7 \%$ of those with a history of defaulting, $15.6 \%$ of those with history of treatment failure, and $16.6 \%$ of those categorised as "others". The likelihood of death was significantly higher among retreatment cases than among the new cases $(\mathrm{X} 2=21.8$; df $=1 ; \mathrm{P}<0.001)$. Regarding treatment regimens, most of the patients $(79.3 \%)$ were on Regimen 1 (2RHZE +4HR; N=1777); while $323(14.4 \%)$ were on Regimen 2 (2RHZES + 1 RHZE $+5 \mathrm{HRE}$ ); 108 (4.8\%) were on Regimen 3 (paediatric
$2 \mathrm{RHZ}+4 \mathrm{HR})$; and $34(1.5 \%)$ were on others treatment, including chemotherapy. There was a significant association between treatment regimen and TB mortality: those on Regimen 2 (re-treatment) were more likely to die than were new cases on Regimen $1(17.2 \%$ vs $11.1 \% ; \mathrm{X} 2=45.7 ; \mathrm{df}=1 ; \mathrm{P}<0.001)$.

Figure 2: TB mortality within the age groups increased with age, with the groups 24 years and below recording less than $10 \%$ mortality rate $(8.4$ among those aged 4 years and below, $4.7 \%$ among those aged 5 to 14 years, and 5.5\% among the 15-24 year group); while those aged 25 years and above had mortality rates higher than $10 \%$ (13.8\% among the 25 to 54 years, $15.7 \%$ among the 55 to 74 year group , and $23.3 \%$ among those older than 75 years. 


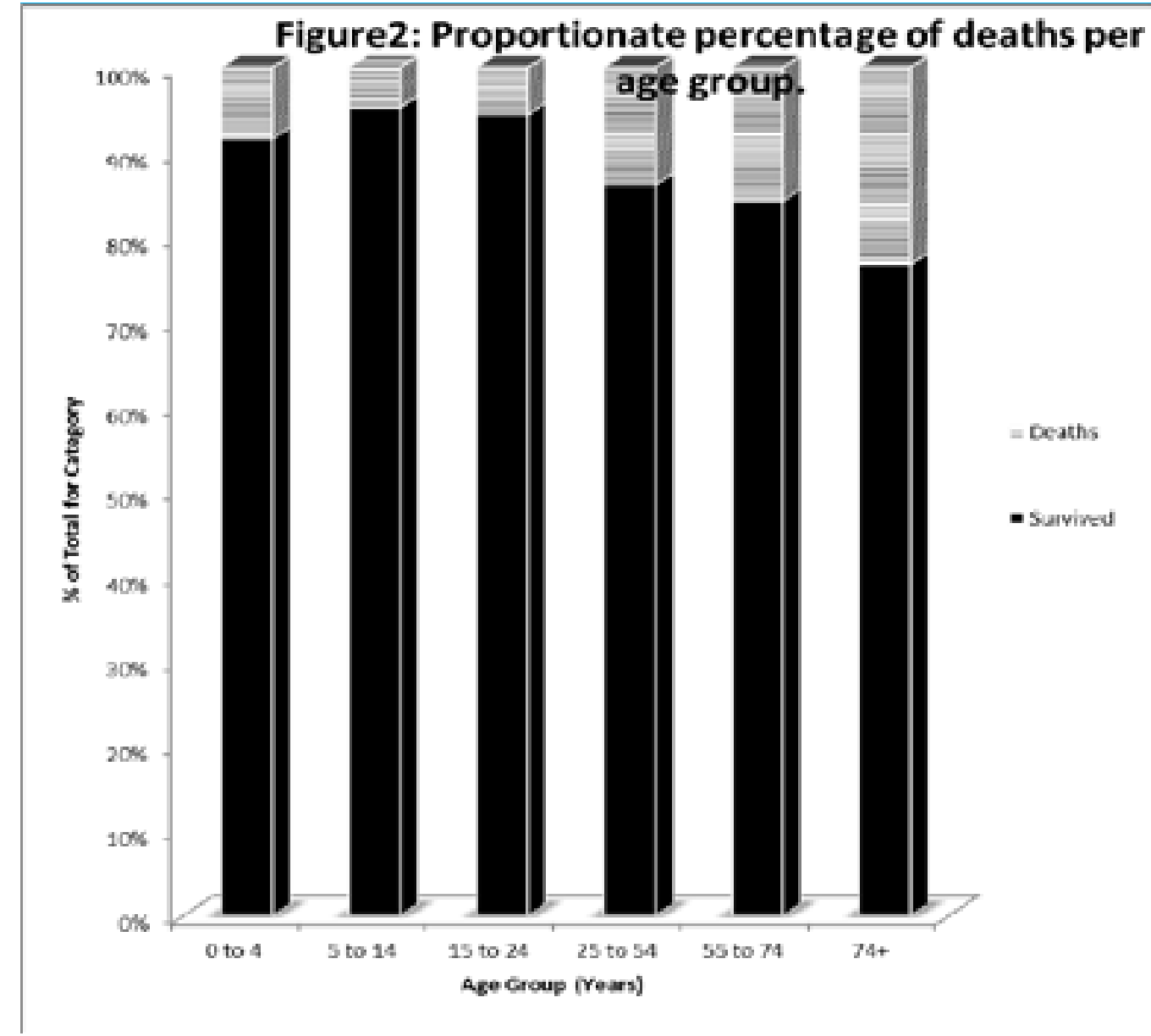

The majority of TB deaths ( $\mathrm{n}=1906,85 \%)$ had in smear positive cases the mycobacteria remained senPTB; , $319(14.2 \%)$ had extra- pulmonary TB; ,15 sitive to the standard regimen for each category.

(0.7\%) had both PTB and EPTB, whilst for two pa- Only 4237 (23.4\%) of the cases recorded in 2008 had tients $(\mathrm{n}=2,0.1 \%)$ the site of disease was not recorded. their HIV status known, and of these $2812(66.4 \%)$ were The mortality rate among those with PTB was $11.9 \%$ HIV positive while $1425(33.6 \%)$ were HIV negative. and 16.1\% among those with EPTB; . While EPTB Among those that were HIV positive, $1362(48.4 \%)$ had contributed to $11.5 \%$ of the recorded TB cases un- smear positive PTB, $379(13.5 \%)$ had smear negative contributed to $16.1 \%$ of the $\mathrm{TB}$, in mortality in that year. , thus patients with EPTB were (13.3\%) had EPTB. Among those that died with known more likely to die than those with PTB $(\mathrm{X} 2=28.8$; HIV status $(\mathrm{n}=557), 83.5 \%(\mathrm{n}=465)$ were HIV posi$\mathrm{df}=1 ; \mathrm{P}<0.001)$ tive, while $92(16.5 \%)$ were HIV negative. The majority of those that died $(n=1746,77.9 \%)$ did not have HIV Among the 15995 PTB patients, $9003(56.3 \%)$ were status recorded, but where it was established the HIV smear positive, $1828(11.4 \%)$ were smear negative positive patients were more likely to die than the HIV and $5164(32.3 \%)$ had no smear results record- negative patients $(\mathrm{P}<0.0001)$. It was not possible ed, for a bacteriological coverage of $73 \%$. Among to disaggregate the data to establish what proportion those with known sputum smear results $(\mathrm{N}=10831)$, of the HIV positive patients that died were on ART or the mortality rate was 17\% among smear negative pa- CPT, and those that had had CD4 tests done.

tients, compared to $13.8 \%$ among smear positive patients. There was higher mortality among smear negative PTB patients than among the smear positive ones $(\mathrm{X} 2=12.5 ; \mathrm{df}=1 ; \mathrm{P}<0.001)$. Among smear positive patients that died, there was no apparent difference in sputum conversion rates between new smear positive and retreatment smear positive patients, suggesting that

Limpopo Province recorded a TB mortality rate of $12.4 \%$ for the year under review, with the majority of deaths recorded among the economically active age group (25-54 years). Mortality was significantly associated with older age, extra pulmonary site of disease,
HIV co- infection; smear negative PTB and previous history of TB. These findings are consistent with previous reports on higher risk of death associated with negative sputum smear, HIV co-infection and other comorbidities, and older age $e^{5-8}$.

The association between HIV infection and TB mortality was evident in the present study: HIV positive patients were more likely to have EPTB and more likely to be smear negative, and mortality was higher among those who were HIV positive. Unfortunately, HIV counselling and testing (HCT) was done in less than a quarter of the patients that started treatment that year, hence the full impact of HIV infection on TB mortality in this setting HIV integrated services, it is apparen from the findings here-in presented that HCT and/or uptake of HIV testing for TB patients may not have been as widespread as envisaged by established guidelines. Higher TB mortality among those who are HIV infected has been reported by others ${ }^{9-11}$. The low levels of HIV testing in TB patients means that many of those who may benefit from HAART are missed out, and yet HAART has been shown to improve treatment outcomes in HIV infected persons with $\mathrm{TB}^{12}$. Moreover, as suggested by Nahed et al ${ }^{13}, \mathrm{~TB}$ patients with HIV infection may require longer than standard therapy to avoid relapses or treatment failure ${ }^{13}$. Clearly, in order for those who are TB-HIV co-infected to benefit from interventions such as co-trimoxazole preventive therapy (CPT) and HAART as advocated by $\mathrm{WHO}^{1}$, there must be HIV testing among all TB patients and intensified case-finding for TB among people living with HIV. Efforts in this regard need to be strengthened in the Limpopo Programme in order for the province to meet the global targets of all TB patients tested for HIV, and all TB patients living with HIV provided with and antiretroviral therapy, and isoniazid preventive therapy for HIV positive people without active TB

There were more males among those that died, which is explicable from the larger number of males among the recorded patients for the year under review. Similarly, the highest number of TB patients recorded was in the age group 25-54 years, and so were the majority of those who died. This age group is the most economically active population, so such high morbidity and mortality has to be addressed to mitigate the possible negative impact on human development. The 2554 year age group is also the one most affected by HIV infection, and it is likely that TB-HIV co-infec- n is partly responsible for both the high morbidity mortality in that age group. The preponderance of new cases in the province, and among those who died, suggests measures to curb TB transmission were not effective, and that the population was not sensitized to seek treatment early.

Patients that were on retreatment had higher mortality than the new cases, which is consistent with findings from Kwa-Zulu Natal ${ }^{14}$. Indeed the retreatment regimen remains of questionable efficacy ${ }^{14,15}$. The programmatic implication of this finding is that strategies such as DOTS should be promoted and adherence enforced to avoid default and treatment failure which necessitate retreatment with less effective regimens.

The lack of data on smear grading and culture and sensitivity, the high number of PTB cases with no smear results $(32.4 \%)$, and the low rate of HIV testing were limitations of the study. The lack of microbiological data is not unique to the Limpopo TB Control Programme, similar observations have been made by others ${ }^{8,11}$; indeed, Komati et al view this as an Africawide problem ${ }^{11}$. Limpopo-specific challenges often include systemic reasons such as sputum not collected for diagnosis, and inaccessibility of laboratory diagnostic facilities in the resource constrained settings many of the facilities operate under.

On the basis of the above findings, the Limpopo TB Control Programme should intensify efforts to offer HIV counselling and testing to all TB patients, and to screen all HIV/AIDS patients for TB, in line with the policy of the South African National Department of Health and Limpopo Province Department as well. The overarching recommendation is for the strengthening of the integrated management of TB and HIV patients, which would cover all the options such as early commencement of ART regardless of CD4 count in those with TB-HIV co-infection and CPT.

\section{Conclusion}

There was higher TB mortality among the economically active age group and older patients, among those who were HIV positive, those that had smear negative PTB, those with EPTB, and among those on retreatment than for the other categories of TB patients. HIV testing was fairly low for the TB patients, which suggests that there is need to intensify HIV testing in TB patients in order to offer supportive therapy such as CPT and ART. 
Acknowledgements: The study was supported by USAID through the Desmond Tutu TB Centre, University of Stellenbosch coordinated Operations Research Assistance Project (ORAP). We thank Mr MM Gafar for his assistance in data collection, and the anonymous AHS reviewers for their helpful comments.

\section{References}

1. World Health Organization (WHO). Global Tuberculosis Control: WHO report 2011. http:// www.who.int/tb/publications/global_report/en/ (accessed12 April 2013).

2. World Health Organization (WHO). Global tuberculosis Control: Epidemiology, Strategy, Financing. Geneva, 2009; pp. 6-33.

3. Abdool-Karim SSA, Chruchyard GJ, Abdool-Karim QA and Lawn SD (2009) HIV infection and tuberculosis in South Africa: an urgent need to escalate the public health response. Lancet, 2009; 374: 921-933.

4. Jeena PM, Pillay P, Pillay T, Coovadia HM. Impact of HIV-1 co-infection on presentation and hospital-related mortality in children with culture proven pulmonary tuberculosis in Durban, South Africa. International Journal of Tuberculosis and Lung Diseases, 2002;6:672-678.

5. Rao VK, Iademarco EP, Fraser VJ, Kollef MH. The impact of comorbidity on mortality following in-hospital diagnosis of tuberculosis. Chest, 1998; 114:1244-52.

6. Hansel NN, Albert W. Wu AW, Betty Chang B, Diette GB. Quality of life in tuberculosis: Patient and provider perspectives. Quality of Life Research, 2004; 13: 639-652. 7. Silva DR, Menegotto DM, Schulz LF, Gazzana MB, Dalcin Pde T. Factors associated with mortality in hospitalized patients with newly diagnosed tuberculosis. Lung, 2010;188:33-41.

8. Peter JG, Theron G, Singh N, Singh A, Dheda K. Sputum induction to aid the diagnosis of smear-nega- tive or sputum-scarce TB in adults from a HIV-endemic setting. European Respiratory Journal, 2013; March 21 (Epub ahead of print).

9. Badri M, Ehrlich R, Wood R, Pulerwitz T, Maartens G. Association between tuberculosis and HIV disease progression in a high tuberculosis prevalence area. International Journal of Tuberculosis and Lung Diseases, 2001; 5:225-232.

10. Whalen CC, Nsubuga P, Okwera A, Johnson JL, Hom DL, Michael NL, et al. Impact of pulmonary tuberculosis on survival of $\mathrm{HIV}$-infected adults: a prospective epidemiologic study in Uganda. AIDS, 2000; 14:1219-1228.

11. Komati S, Shaw PA, Stubbs N, Mathibedi MJ, Malan L, Sangeni P, Metcalf JA, Masun H, Hassim S. Tuberculosis risk factors and mortality for HIV-infected persons receiving antiretroviral therapy in South Africa. AIDS, 2010, 24:1849-1855

12. Straetemans M, Bierrenbach AL, Nagelkerke N, Glaziou P, van der Werf MJ (2010) The Effect of Tuberculosis on Mortality in HIV Positive People: A Meta-Analysis. PLoS One, 5(12): e15241. doi:10.1371/ journal.pone.0015241

13. Nahed P, Leah C. Gozelez T. Treatment outcome of TB patient co-infected with HIV. American Journal of Respiratory and Critical Care Medicine, 2006; 3: 1199-1206 14. Schreiber YS, Herrera AF, Wilson D, Wallengren K, Draper R, Muller J, Dawood H, Doucette S, Cameron DW, Alvarez GG.Tuberculosis retreatment category predicts resistance in hospitalized retreatment patients in a high HIV prevalence area. International Journal of Tuberculosis and Lung Diseases, 2009; 13:1274-80.

15. López E, Ayakaka I, Levin J, Reilly N\& Mumbowa F (2011). Effectiveness of the Standard WHO Recommended Retreatment Regimen (Category II) for Tuberculosis in Kampala, Uganda: A Prospective Cohort Study. PLoS Medicine, 8(3): e1000427. 